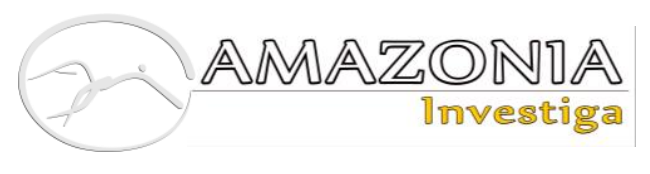

DOI: https://doi.org/10.34069/AI/2021.48.12.7

How to Cite:

Cantillo Lores, A.R., Guerra Cleger, Y., Vistel, M., Bosch Escobar, J., \& Chil Núñez, I. (2021). Estabilidad de la formulación sales de rehidratación oral de osmolaridad reducida (polvo y solución oral). Amazonia Investiga, 10(48), 63-73. https://doi.org/10.34069/AI/2021.48.12.7

\title{
Estabilidad de la formulación sales de rehidratación oral de osmolaridad reducida (polvo y solución oral)
}

\section{Formulation stability oral rehydration salts of reduced osmolarity (powder and oral solution)}

\section{Estabilidade da formulação de sais de reidratação oral de osmolaridade reduzida (pó e solução oral)}

\author{
Written by: \\ Ana Rosa Cantillo Lores $^{22}$ \\ https://orcid.org/0000-0003-2143-1363 \\ Yadira Guerra Cleger ${ }^{23}$ \\ https://orcid.org/0000-0001-9671-6684 \\ Marlen Vistel ${ }^{24}$ \\ https://orcid.org/0000-0002-4124-2511 \\ Jorge Bosch Escobar ${ }^{25}$ \\ https://orcid.org/0000-0002-8445-6616 \\ Idelsy Chil Núñez ${ }^{26}$ \\ https://orcid.org/0000-0003-4661-0472
}

\section{Resumen}

Las Sales de Rehidratación Oral de Osmolaridad Reducida son efectivas para tratar las enfermedades diarreicas siendo una importante herramienta en la prevención de muertes y comorbilidades. El estudio realizado tuvo como propósito evaluar la estabilidad en estante de la formulación en forma de polvo y a la solución oral a través de controles físicos, químicos y microbiológicos, basado en la directiva de estabilidad vigente en Cuba. La presentación de las muestras en polvo es en sobres de poliésteraluminio polietileno por 20,5 g, y su almacenamiento a $30 \pm 2{ }^{\circ} \mathrm{C}$ a una humedad relativa de $70 \pm 5 \%$. Las mismas se evalúan por un año con una frecuencia trimestral a partir de su fecha de fabricación. La solución oral se prepara en frascos cónicos esterilizados, la que se conserva entre $30 \pm 20 \mathrm{C}$ en la primera hora y a $2-8$ 0C hasta las 24 horas posteriores a su

\begin{abstract}
The Oral Rehydration Salts of Reduced Osmolarity are effective to treat diarrheal diseases being a strong tool for the prevention of deaths and comorbidities. The purpose of the study was to evaluate the shelf stability of the formulation in powder form and the oral solution through physical, chemical and microbiological controls, based on the directive stability current in Cuba. The presentation of de powder samples is in polyester-aluminum polyethylene envelopes for $20,5 \mathrm{~g}$ and stored at $30 \pm 2{ }^{\circ} \mathrm{C}$ at a relative humidity of $70 \pm 5 \%$ and checked at a frequency of $0,3,6,9$ and 12 months from the date of manufacture of the powdered product. They are evaluated for a year with a quarterly frequency from their date of manufacture. The oral solution is prepared in sterilized conical bottles, which is kept between $30 \pm 20 \mathrm{C}$ within the 1 st hour and 2 - 8 0C until 24 hours after its preparation. The
\end{abstract}

\footnotetext{
${ }^{22}$ Ingeniera Química. Master en Ingeniería Química. Empresa Laboratorio Farmacéutico Oriente. Tecnólogo A de Procesos Industriales, Santiago de Cuba, Cuba.

${ }^{23}$ Licenciada en Ciencias Farmacéuticas. Optando por el título de Master en Servicios Farmacéuticos. Empresa Laboratorio Farmacéutico Oriente. Tecnólogo A de Procesos Industriales, Santiago de Cuba, Cuba.

${ }^{24}$ Licenciada en Química. Máster en Medicina Natural Tradicional. Empresa Laboratorio Farmacéutico Oriente. Tecnólogo A de Procesos Industriales, Santiago de Cuba, Cuba.

${ }^{25}$ Técnico en Química Industrial. Empresa Laboratorio Farmacéutico Oriente. Tecnólogo de Procesos Industriales, Santiago de Cuba, Cuba.

${ }^{26}$ Doctora en Ciencias de la Salud. Profesora Titular. Departamento de Farmacia. Universidad de Oriente, Santiago de Cuba. Cuba.
} 
preparación. Las especificaciones evaluadas se determinan según las Farmacopeas de Estados Unidos Ed. 40, 2017 y Británica 2013, empleando métodos analíticos validados. Se demuestra que estos parámetros en las muestras en polvo se mantienen dentro de los límites de calidad establecidos, y que los resultados hallados para las determinaciones cuantitativas presentan una distribución normal, por lo que se establece que el proceso se encuentra en control estadístico con un nivel de confianza del $95 \%$, lo que es extensivo a la solución oral, razones por las que se concluye que el producto posee una adecuada estabilidad en las condiciones estudiadas, garantía de su seguridad y eficacia farmacológica.

Palabras claves: Enfermedad diarreica aguda, Estudio de estabilidad, Osmolaridad reducida, Sales de Rehidratación Oral, Soluciones para rehidratación oral. evaluated specifications are determined according to United States pharmacopoeias USP40, 2017 and British BP 2013 and using validated analytical methods. It is demonstrated that these parameters in the powdered samples are kept within the established quality limits and that the results found for a quantitative determinations present a normal distribution, for which it is established that the process is in statistical control with a confidence level of $95 \%$, which is extensive to the oral solution, reasons for which it is concluded that the product has adequate stability under the conditions studied, guarantee of safety and pharmacological efficacy.

Key Words: Acute diarrheal disease, Stability of study, Reduced Osmolarity, The Oral Rehydration Salts, Oral Rehydration Solutions.

\section{Resumo}

Os Sais de Reidratação Oral de Osmolaridade Reduzida são eficazes no tratamento de doenças diarreicas sendo uma importante ferramenta na prevenção de óbitos e comorbidades. O objetivo do estudo realizado foi avaliar a estabilidade de prateleira da formulação em pó e da solução oral por meio de controles físicos, químicos e microbiológicos, com base na atual diretriz de estabilidade de Cuba. As amostras de pó são apresentadas em sachês de poliéster-alumínio polietileno de $20,5 \mathrm{~g}$, e seu armazenamento a $30 \pm 2^{\circ} \mathrm{C}$ e umidade relativa de $70 \pm 5 \%$. São avaliados por um ano com periodicidade trimestral a partir da data de fabricação. A solução oral é preparada em frascos cônicos esterilizados, que são armazenados entre $30 \pm 2^{\circ} \mathrm{C}$ na primeira hora e $2-80 \mathrm{C}$ até 24 horas após o seu preparo. As especificações avaliadas são determinadas de acordo com as Farmacopeias dos estados Unidos Ed. 40 de 2017 e a Britânica de 2013 usando métodos analíticos validados. Se demonstra que estes parâmetros nas amostras de pó se mantêm dentro dos limites de qualidade estabelecidos, e que os resultados encontrados para os de ordem quantitativa apresentam distribuição normal, portanto fica estabelecido que o processo está sob controle estatístico com $95 \%$ de confiança nível, extensivo à solução oral, motivos pelos quais se conclui que o produto apresenta estabilidade adequada nas condições estudadas, garantia da sua segurança e eficácia farmacológica.

Palavras-chave: Doença diarreica aguda; Estudo de estabilidade; Osmolaridade reduzida; Sais de reidratação oral; Soluções de reidratação oral.

\section{Introducción}

Las enfermedades diarreicas agudas constituyen un serio problema de salud que enfrentan hoy los países subdesarrollados y algunos desarrollados en el mundo, siendo una de las principales causas de morbilidad y mortalidad (Galván, 2014). Estudios realizados en la Sociedad latinoamericana de infectología pediátrica en el 2015, estimó más de 1.3 millones de muertes por lo que se define como la cuarta causa de muerte en niños menores de cinco años (Herrera, García y Mascareñas, 2018), mientras en el 2020 constituye la segunda causa de muerte afectando a 525000 niños cada año y aunque se observa un decrecimiento en sus cifras es considerada como un relevante problema de salud pública en todas las edades. Esta disminución puede estar relacionada con los determinantes sociales de la salud en los países en desarrollo y avances en la investigación (Vásquez y Arias, 2020).

En Cuba la mortalidad por esta causa es mínima y la morbilidad es comparable a los países desarrollados, tanto en atenciones médicas de los menores de cinco años como en los restantes grupos de edad (Blanco y Reyes, 2015); razones por lo que no constituye un importante problema de salud, lo que refleja el excelente trabajo multidisciplinario en el control de factores de riesgo tales como, un estricto control epidemiológico, una vigilancia efectiva y 


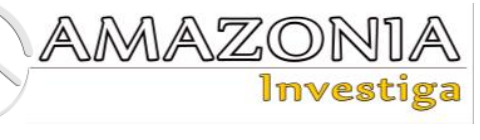

un Sistema de Salud Pública comprometido con la sociedad que ha garantizado que la propagación de esta enfermedad sea minimizada (Povea y Hevia, 2019). Hace más de 25 años se demostró que el suministro conjunto de líquidos y de las Sales de Rehidratación Oral (SRO) resulta una terapia efectiva para la prevención de muertes por esta causa. Luego de extensas investigaciones la Organización Mundial de la Salud (OMS) emitió una nueva fórmula a base de sodio y glucosa para prevenir y/o tratar la deshidratación, con lo que se redujo a un $33 \%$ la necesidad de administrar líquidos intravenosos superando la fórmula anterior (UNICEF, 2001; OPS, 2008).

Por la importancia del uso de las SRO en el tratamiento de enfermedades diarreicas agudas, la Empresa Laboratorio Farmacéutico Oriente (LFO) produce este medicamento hace varios años. Esta fue fundada en 1972 y posee una amplia cartera de productos de excelente prestigio nacional e internacional certificados en buenas prácticas y de elevada calidad garantizada por una fuerza de trabajo calificada y éticamente comprometida; cuyo objeto social es producir y comercializar medicamentos, productos naturales y equipos médicos para contribuir a la salud y bienestar de la sociedad. La planta de tabletas y polvos del LFO es la responsable de producir y tributar al cuadro básico de medicamentos del país el $17 \%$ de los surtidos en forma de tabletas, siendo una de sus principales fortalezas los productos naturales a partir de plantas medicinales, derivados apícolas y otros, aprovechando las propiedades antioxidantes y nutritivas que poseen; y el $100 \%$ de polvos orales como las Sales de Rehidratación Oral que se distribuyen en todo el país. Teniendo en cuenta esto último se pretende incrementar los niveles productivos de esta forma farmacéutica con la puesta en marcha de una nueva planta de sales y polvos orales y con ello lograr cumplir el plan del año con 1412.46 millones de sobres y 6341.662908 millones de pesos, además de satisfacer la demanda anual de este producto con 4.2 millones de sobres, logrando sustituir importaciones.

Para el año 2022 existe la propuesta de incrementar las producciones en aras de lograr un importante y nuevo renglón exportable en nuestra empresa y país. Por ello y siguiendo lo establecido por la OMS se impone el desarrollo de una nueva formulación de SRO de osmolaridad reducida por sus mejores resultados y de menor costo para el país, debido a la sustitución de importaciones y a la disminución en cantidad de dos de sus componentes resultando más efectiva en el cuadro de diarreas y vómitos.

Esta nueva formulación está compuesta por ingredientes farmacéuticos activos cuyo uso se justifica sobre la base de su fácil administración a personas con dificultad para deglutir, es un medio altamente eficaz y su empleo cuenta con una actuación más rápida. Otro argumento a su favor es la rápida absorción que presenta ya que estas se encuentran en solución (Herrera, García y Barrios, 2008; Granda, 2003; Materán, Tomat, Salvatierra, León y Marcano, 2014). Esto demanda la necesidad de asegurar que la calidad de sus elementos precursores sea estable en un sentido absoluto y estricto para asegurar su efectividad y pureza una vez que llegue al paciente; ya que su identidad química, color, consistencia, entre otras características puede cambiar durante el tiempo transcurrido desde su manufactura hasta el momento de su consumo final, le confieren al consumidor la seguridad de que el medicamento se encuentra en condiciones aceptables (Lemus, 2006).

Por ello se deben realizar estudios de estabilidad al producto terminado que permitan el monitoreo de o los principios activos en el tiempo, lo que representa un significativo eslabón en el desarrollo y formulación de toda forma terminada. De esta manera se puede definir las condiciones de almacenamiento en el envase propuesto y establecer el tiempo de vida útil. Estos estudios contemplan la conservación de la potencia, pureza, características organolépticas y otras, que garantizan su efectividad. Esta estabilidad puede verse afectada por diversos factores como son las condiciones ambientales (luz, humedad, temperatura, aire), y factores intrínsecos a la fabricación como el tamaño de partícula, $\mathrm{pH}$, naturaleza del envase y la presencia de otros productos químicos procedentes de contaminación (García, Montes de Oca y Salomón, 2013).

Teniendo en cuenta las consideraciones anteriores, en este trabajo se plantea como objetivo: Evaluar la estabilidad en estante de la formulación de las Sales de Rehidratación Oral de Osmolaridad Reducida, así como de la solución oral en las condiciones propuestas.

\section{Marco Teórico}

La terapia de rehidratación oral se ha convertido en los últimos tiempos en la piedra angular para el tratamiento de las enfermedades diarreicas, constituyendo un gran avance para tratar de forma segura la deshidratación que responde a la 
alteración o perdida de agua y sales minerales en el organismo y que puede estar producida por diarrea de diversas etiologías en todas las edades, excepto cuando la deshidratación es grave. Es un medio altamente eficaz y su empleo cuenta con una actuación rápida (Granda, 2003; Materán, Tomat, Salvatierra, León y Marcano, 2014). Las enfermedades diarreicas agudas se definen como la presencia de heces líquidas o acuosas asociada a un aumento de la frecuencia, al menos 3 deposiciones en 24 horas y pueden ir acompañadas de vómitos y/o fiebres con una duración de hasta 14 días (Pizarro-Torres, 2005). Como resultado de las diferentes pérdidas de electrolitos o por la presencia de agentes infecciosos, ocurre la deshidratación, elemento fundamental de que parte el diseño de las sales de rehidratación oral para el tratamiento de estas afecciones (Ministerio de Salud del Perú, 2010); éstas se presentan en forma de una mezcla uniforme de polvo de color blanco cristalino usado como suplemento para la deshidratación y está compuesto por dextrosa anhidra, cloruro de sodio, citrato de sodio dihidratado y cloruro de potasio (USP 40, 2017).

Se ha demostrado que las altas temperaturas y porcentajes altos de humedad en el ambiente, tal como se presentan en los climas tropicales, facilitan la descomposición de los ingredientes de las SRO, de modo que se alteran su formulación y sus propiedades terapéuticas. EI deterioro del medicamento puede conducir a que no sea usado, aún en los casos en que no haya ocurrido una alteración farmacológica y terapéutica significativa, por lo que resulta conveniente estudiar su estabilidad en condiciones adversas de almacenamiento (In y Miritello, 1988).

Conceptos (Pérez, Saíz y Pérez, 2009).

- La estabilidad es la capacidad de un producto farmacéutico para conservar sus propiedades químicas, físicas, microbiológicas y biofarmacéuticas dentro de límites especificados a lo largo de su tiempo de conservación

- Estudios de estabilidad en tiempo real (a largo plazo o vida estante) son los experimentos relacionados con las características físicas, químicas, biológicas, biofarmacéuticas y microbiológicas de un medicamento, durante y más allá del tiempo de conservación y el periodo de almacenamiento previstos, y que se hacen en muestras mantenidas en condiciones de almacenamiento semejantes a las que habrá en el mercado al que van destinadas. Los resultados se utilizan para determinar el tiempo de conservación, confirmar este y recomendar las condiciones de almacenamiento.

- Fecha de caducidad es la que se inscribe en el recipiente individual de un producto medicamentoso (generalmente en la etiqueta) y que precisa el momento hasta el cual el producto se ajusta a sus especificaciones, siempre y cuando se haya almacenado correctamente. Se establece para cada lote agregando el tiempo de conservación a la fecha de fabricación.

- Período de utilización es el tiempo durante el cual puede usarse una preparación reconstituida o la forma farmacéutica acabada que viene en un recipiente abierto de dosis múltiples.

\section{Metodología}

Se realizó un estudio de estabilidad en condiciones de estante, experimental y prospectivo del producto Sales de Rehidratación Oral Osmolaridad reducida. La investigación se llevó a cabo en la Empresa Laboratorio Farmacéutico Oriente de Santiago de Cuba en el período comprendido desde marzo del 2018 hasta marzo del 2019.

Para la elaboración de la formulación en estudio se utilizaron materias primas suministradas por la Empresa Importadora y Exportadora FARMACUBA las que fueron analizadas siguiendo las monografías oficiales de las Farmacopeas de Estados Unidos o Británica según corresponda, para comprobar su calidad.

En el procesamiento estadístico se realizó un análisis donde se calcularon la media, la desviación estándar y el coeficiente de variación de los datos obtenidos para cada muestra. Se determinó el valor del sesgo estandarizado y la Curtosis estandarizada indicativo de desviaciones significativas de la normalidad, a partir de los cuales se define si las muestras tienen una distribución normal; así como el error, que muestra el grado de desviación de la precisión de los ensayos realizados. Los datos fueron procesados utilizando el paquete estadístico Statgraphics Centurion XV.II.

Descripción de la composición del producto utilizado: cada sobre de 20,5 g contiene: 
Tabla 1.

Composición de las Sales de Rehidratación Oral de Osmolaridad Reducida.

\begin{tabular}{lll}
\hline Nombres químicos & Componentes de la formulación & Fabricante/ País \\
\hline Dextrosa Anhidra & D-glucosa & Shangxue Glucose / China \\
Cloruro de Sodio & Cloruro de Sodio & DC Fine Chemicals / España \\
Citrato de Sodio dihidratado & Sal trisódica del ácido & DC Fine Chemicals / España \\
Cloruro de potasio & Cloruro de potasio & Hebei Huachen Pharmaceutical \\
\hline
\end{tabular}

Fuente: Elaborada por los autores

Para el envase se utilizaron sobres de papel aluminio-poliéster-polietileno por 20,5 g. Una vez propuesta la formulación se procedió a la elaboración de tres lotes pilotos a escala industrial de 7317 sobres en la Planta 1 de tabletas y Polvos del Laboratorio Farmacéutico Oriente, identificados con los números de lote 8001L, 8002L y 8003L.

\section{Estudio de estabilidad}

Para realizar el estudio de estabilidad de los lotes elaborados se realizan los análisis físicoquímico, químico y microbiológico de las muestras seleccionadas empleando la monografía recogida en la Farmacopea de los Estados Unidos de América, Ed.40, 2017 (USP 40, 2017), aunque se realiza la determinación del contenido de citrato por la Farmacopea Británica Ed. 7th, 2013 (BP 2013), teniendo en consideración los requerimientos del Centro de Control Estatal de Medicamentos (CECMED) para los estudios de estabilidad (Regulación No. 23-2000).

Las muestras en polvo en el envase propuesto son almacenadas a una temperatura de $30 \pm 2{ }^{\circ} \mathrm{C}$, y una humedad relativa de $70 \pm 5 \%$, las mismas se revisan con una frecuencia trimestral $(0,3,6,9 \mathrm{y}$ 12 meses) a partir de su fecha de fabricación. La solución oral es almacenada entre $30 \pm 2{ }^{\circ} \mathrm{C}$ en la primera hora de preparada, y a $2-8{ }^{\circ} \mathrm{C}$ hasta las 24 horas posteriores, estudio que simula las condiciones a las que es almacenada esta solución en el hogar.

\section{Ensayos físico-químicos que se determinan al polvo oral:}

Característica organoléptica: observe debajo de una lámpara fluorescente una muestra previamente seleccionada de la mezcla, verifique su color, apariencia y presencia de impurezas.

Criterio de aceptación: polvo blanco cristalino, libre de pintas e impurezas mecánicas.
Determinación de pH: este ensayo se realiza con la solución oral. Para ello se disuelve un sobre de $20.5 \mathrm{~g}$ en un litro de agua destilada.

Una vez preparado el material de prueba enjagüe el sensor de $\mathrm{pH}$ con agua. Luego encienda el pH metro. Pasado unos minutos proceda a ajustar el equipo con las soluciones buffers y después enjagüe con la solución de ensayo. Sumerja el sensor de $\mathrm{pH}$ en la muestra y registre la lectura obtenida.

\section{Criterio de aceptación: 7,0 - 8,8}

Pérdida por desecación: conecte la estufa y gradúela a $50{ }^{\circ} \mathrm{C}$ y espere hasta que alcance la temperatura. Una vez logrado, coloque dentro de ella los pesafiltros y sus tapas por un espacio de 30 minutos, enfríelos tapados en una desecadora con silica gel a temperatura ambiente y pasado los 15 minutos pese con exactitud cada uno. Reduzca el tamaño de las partículas de la muestra hasta aproximadamente $2 \mathrm{~mm}$, y transfiera con rapidez a los pesafiltros tarados $\left(\begin{array}{lll}1 & \text { o } & \mathrm{g} \text { de }\end{array}\right.$ polvo), distribuya cuidadosamente la muestra tan uniformemente como sea posible, mezclando suavemente hacia los lados. La profundidad debe ser de aproximadamente $5 \mathrm{~mm}$ y pese. Coloque los pesafiltros destapados y sus tapas en la estufa por dos horas, enfríe estos tapados, y halle su masa. Vuelva a colocarlos en la estufa por una hora. Este proceso se repite hasta obtener pesadas constantes que no difieran en más de $0,50 \mathrm{mg}$ por $\mathrm{g}$ de sustancia tomada, realizando la segunda pesada después de una hora adicional de secado.

Criterio de aceptación: La pérdida obtenida no es mayor que un $1,0 \%$.

\section{Determinación de los ensayos químicos}

Dextrosa: El contenido de los envases en dosis única se tritura previamente a polvo fino, y se transfiere un equivalente a $20 \mathrm{~g}$ de dextrosa a un matraz volumétrico de $100 \mathrm{~mL}$, se diluye con agua hasta volumen y se mezcla (solución madre de Dextrosa). 
Mida exactamente $50 \mathrm{~mL}$ de la solución anterior y transfiera a un matraz de un trazo de $100 \mathrm{~mL}$, adicione $0,2 \mathrm{~mL}$ de solución de hidróxido de amonio $6 \mathrm{~N}$, lleve a volumen con agua y mezcle. Encienda el polarímetro y pasados los $30 \mathrm{~min}$ determine la rotación angular de la solución en un tubo polarimétrico adecuado y realice los cálculos necesarios.

\section{Criterio de aceptación: 90,0\%-110,0\%}

\section{Sodio y potasio}

Preparación de la solución madre de sodio: Mida cuidadosamente de la solución madre de Dextrosa (véase determinación de Dextrosa) un volumen equivalente a $0,23 \mathrm{mg} / \mathrm{mL}$ de sodio $\mathrm{y}$ transfiera a un matraz volumétrico de $1000 \mathrm{~mL}$, diluya con agua a volumen y mezcle.

Preparación de la solución madre de potasio: Mida cuidadosamente de la solución madre de Dextrosa (véase determinación de Dextrosa) un volumen equivalente a $0,39 \mathrm{mg} / \mathrm{mL}$ de potasio $\mathrm{y}$ transfiera a un matraz volumétrico de $200 \mathrm{~mL}$, diluya con agua a volumen y mezcle.

Solución muestra de sodio: Transfiera un volumen equivalente $0,0115 \mathrm{mg} / \mathrm{mL}$ de sodio, a partir de la solución madre de sodio a un matraz de un trazo de $25 \mathrm{~mL}$, diluya con solución diluyente de litio (Disuelva $1.04 \mathrm{~g}$ de nitrato de litio a un matraz de un trazo de $1000 \mathrm{ml}$, adicione agente tensoactivo, complete con agua a volumen y mezcle)

Solución muestra de potasio: Transferir un volumen equivalente a $0,01955 \mathrm{mg} / \mathrm{mL}$ de potasio a partir de solución madre de potasio a un matraz de un trazo de $25 \mathrm{~mL}$, completar a volumen con solución diluyente de litio $\mathrm{y}$ mezcle.

Determinación: Encienda el fotómetro de llama y llevar todos los botones a cero, seleccionar el filtro de sodio y ajustar el cero con la solución diluyente de litio y a 10 con la solución de referencia de sodio y potasio. Mida las lecturas de emisión correspondiente al sodio en la solución muestra de sodio luego pasar agua 2 o 3 minutos para limpiar las tuberías del equipo.

Seleccionar el filtro de potasio y ajustar el cero con la solución diluyente de litio y a 20 con la solución de referencia de sodio y potasio. Medir las lecturas de emisión correspondiente al potasio en la solución muestra de potasio luego pasar agua 2 o 3 minutos al finalizar para limpiar las tuberías del equipo y realice los cálculos correspondientes.

Criterio de aceptación: Potasio y sodio: 90,0\%$110,0 \%$ de la cantidad declarada.

Cloruros: Mida exactamente un volumen equivalente a $55 \mathrm{mg}$ de cloruro de la solución madre dextrosa y transfiera a un recipiente adecuado. Valorar de modo directo con la Solución volumétrica de Nitrato de plata $0,1 \mathrm{~N}$ SV hasta que el cloruro de plata flocule y la mezcla adquiera un ligero color rosado, usando cromato de potasio SR como indicador. Calcule el porcentaje de la cantidad declarada de cloruro en el envase de dosis única.

Detección del punto final: Visual

Criterio de aceptación: $90,0 \%-110,0 \%$ de la cantidad declarada (USP 40, 2017).

Citrato: Disperse $2,8 \mathrm{~g}$ de polvo en $80 \mathrm{~mL}$ de ácido acético glacial anhidro y mezcle suavemente para lograr homogeneidad en la mezcla, caliente la misma aproximadamente a 50 ${ }^{0} \mathrm{C}$ y luego deje enfrié. Transfiera la mezcla obtenida a un matraz de un trazo de $100 \mathrm{~mL}$, complete a volumen con ácido acético glacial anhidro, mezcle, y deje reposar por 10 minutos. Mida cuidosamente $20 \mathrm{~mL}$ del líquido sobrenadante y transfiera a un frasco cónico, adicione solución indicadora de 1naftolbenceina, mezcle y tape con papel aluminio. Pinche el papel de aluminio con la punta de la bureta y valore con solución volumétrica de ácido perclórico y agitación magnética hasta obtener color verde esmeralda. Realice los cálculos correspondientes (BP, 2013).

Criterio de aceptación: 90,0\%-110,0\% de la cantidad declarada (BP, 2013).

\section{Ensayo microbiológico. Método de Vertido en Placa-Para placas de Petri}

Disolver $10 \mathrm{~g}$ del producto a examinar en Solución Amortiguada de Fosfato de pH 7,2.

Conteo total de microorganismos aeróbicos: Agregar a dos placas $1 \mathrm{~mL}$ de la muestra preparada y adicione a cada una de 15 a $20 \mathrm{~mL}$ de medio Triptona Soya Agar (TSA) previamente fundido y enfriado manteniendo la temperatura aproximadamente a $45^{\circ}$. Mezclar en ambas placas las muestras y dejar solidificar el medio. Incubar las placas de $(30 \text { a } 35)^{\circ} \mathrm{C}$ de 3 a 5 días. 


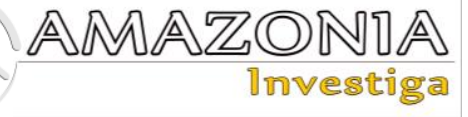

Conteo de hongos y bacterias: Agregar a dos placas $1 \mathrm{~mL}$ de la muestra preparada y adicione a cada una de 15 a $20 \mathrm{~mL}$ de medio Sabouraud Dextrosa Agar (SDA) previamente fundido $\mathrm{y}$ enfriado manteniendo la temperatura aproximadamente a $45^{\circ} \mathrm{C}$. Mezclar en ambas placas las muestras y dejar solidificar el medio. Incubar las placas de $(20 \text { a } 25)^{\circ} \mathrm{C}$ durante 7 días.

Interpretación de los resultados: Pasado estos días realice el conteo total de hongos y bacterias en las placas. Si no existe crecimiento microbiano tome el resultado como $<10$ U.F.C

Ensayos físico-químicos que se determinan a la solución oral: para las determinaciones se envasa la solución en frascos transparentes de vidrio (borosilicato) previamente esterilizados.

\section{Determinación de pH}

Criterio de aceptación: 7,0 - 8,8 (USP 40, 2017) Formación de precipitado: disuelva el contenido del sobre en un litro de agua.
Criterio de aceptación: el contenido se disuelve completamente sin formación de precipitado.

Claridad de la solución: disuelva 5 gr en 90 mL de agua libre de dióxido de carbono y complete a 100 $\mathrm{mL}$ con el mismo solvente. Se realiza una inspección visual.

Criterio de aceptación: Solución transparente, clara e incolora. No presenta pintas ni impurezas mecánicas (BP, 2013).

\section{Ensayo microbiológico por el Método de Vertido en Placa-Para placas de Petri (USP 40, 2017). Resultados}

Ensayos físicos, químicos y microbiológicos que se determinan a la formulación de Sales de Rehidratación Oral Osmolaridad reducida polvo oral.

La tabla que se muestra a continuación presenta los resultados obtenidos en los ensayos físicoquímicos, químicos y microbiológicos que se realizaron al polvo oral en los 12 meses de estudio.

Tabla 2.

Resultados de los ensayos físico-químicos, químicos y microbiológicos realizados a las Sales de Rehidratación Oral Osmolaridad reducida (polvo oral).

\begin{tabular}{lllllll}
\hline Índices & Lotes & Meses & & & & \\
\cline { 3 - 7 } & & 0 & 3 & 6 & 9 & 12 \\
Descripción & $8001 \mathrm{~L}$ & Responde & Responde & Responde & Responde & Responde \\
& $8002 \mathrm{~L}$ & Responde & Responde & Responde & Responde & Responde \\
& $8003 \mathrm{~L}$ & Responde & Responde & Responde & Responde & Responde \\
pH & $8001 \mathrm{~L}$ & 8,22 & 7,85 & 7,9 & 8,31 & 8,19 \\
$\mathbf{( 7 , 0 - 8 , 8 )}$ & $8002 \mathrm{~L}$ & 7,83 & 7,96 & 7,85 & 8,10 & 8,20 \\
Pérdida por & $8003 \mathrm{~L}$ & 8,06 & 8,0 & 8,2 & 8,8 & 8,16 \\
Desecación & $8001 \mathrm{~L}$ & 0,02 & 0,45 & 0,35 & 0,23 & 0,4 \\
(\%) & $8002 \mathrm{~L}$ & 0,18 & 0,5 & 0,3 & 0,34 & 0,45 \\
& $8003 \mathrm{~L}$ & 0,4 & 0,4 & 0,3 & 0,35 & 0,5 \\
Sodio total (\%) & $8001 \mathrm{~L}$ & 102,9 & 102,9 & 104,4 & 97.8 & 100,1 \\
& $8002 \mathrm{~L}$ & 103,9 & 103,9 & 104,4 & 100,2 & 102,1 \\
Potasio (\%) & $8003 \mathrm{~L}$ & 102,9 & 102,9 & 102,9 & 97,8 & 101,5 \\
& $8001 \mathrm{~L}$ & 100,5 & 102,3 & 96,6 & 96,8 & 94,3 \\
Citratos (\%) & $8002 \mathrm{~L}$ & 100,8 & 102,7 & 100,0 & 96,3 & 94,4 \\
& $8003 \mathrm{~L}$ & 99,6 & 102,3 & 94,3 & 95,8 & 94,8 \\
& $8001 \mathrm{~L}$ & 99,7 & 97.7 & 101,1 & 99,2 & 98,5 \\
Cloruro (\%) & $8002 \mathrm{~L}$ & 95,1 & 102,5 & 105,3 & 97,4 & 102,2 \\
& $8003 \mathrm{~L}$ & 104,3 & 104,3 & 106,5 & 97,4 & 100,7 \\
& $8001 \mathrm{~L}$ & 103,1 & 100,5 & 99,1 & 96,2 & 95,8 \\
Dextrosa (\%) & $8002 \mathrm{~L}$ & 103,7 & 101,6 & 95,0 & 96,7 & 95,0 \\
& $8003 \mathrm{~L}$ & 102,3 & 99,0 & 94,4 & 98,3 & 93,7 \\
Conteo & $8001 \mathrm{~L}$ & 98,0 & 97.0 & 97,2 & 96,8 & 97,8 \\
Microbiano & $8002 \mathrm{~L}$ & 98,9 & 97.5 & 98,4 & 100,7 & 99,2 \\
& $8003 \mathrm{~L}$ & 100,4 & 99,3 & 96,1 & 98,2 & 99,1 \\
\hline & $8001 \mathrm{~L}$ & Responde & Responde & Responde & Responde & Responde \\
& $8002 \mathrm{~L}$ & Responde & Responde & Responde & Responde & Responde \\
& $8003 \mathrm{~L}$ & Responde & Responde & Responde & Responde & Responde \\
\hline
\end{tabular}

Fuente: Elaborada por los autores

Los resultados obtenidos en los lotes estudiados (Tabla 2) muestran que las especificaciones de calidad evaluadas se mantienen dentro de sus criterios de aceptación a lo largo de los 12 meses. 
Tabla 3.

pH. Procesamiento estadístico.

\begin{tabular}{llll}
\hline Lotes & $\mathbf{8 0 0 1} \mathbf{~ L}$ & $\mathbf{8 0 0 2} \mathbf{L}$ & $\mathbf{8 0 0 3} \mathbf{L}$ \\
\hline Media de proceso & 8.09 & 7.99 & 8.26 \\
Desviación Estándar & 0,21 & 0,13 & 0,38 \\
Coeficiente de Variación (\%) & 2,76 & 1,92 & 4,80 \\
Error Estándar & 0,11 & 0,077 & 0,19 \\
Sesgo Estandarizado & 0,16 & $-0,06$ & 1,39 \\
Curtosis Estandarizada & $-1,79$ & $-0,84$ & 1,31 \\
\hline
\end{tabular}

Fuente: Elaborada por los autores

Tabla 4

Pérdida por secado. Estadística.

\begin{tabular}{llll}
\hline Lotes & $\mathbf{8 0 0 1} \mathbf{~ L}$ & $\mathbf{8 0 0 2} \mathbf{~}$ & $\mathbf{8 0 0 3} \mathbf{L}$ \\
\hline Media de proceso & 0,31 & 0,35 & 0,39 \\
Desviación Estándar & 0,09 & 0,15 & 0,07 \\
Coeficiente de Variación $(\%)$ & 26,38 & 23,45 & 22,04 \\
Error Estándar & 0,05 & 0,05 & 0,04 \\
Sesgo Estandarizado & $-0,78$ & 0,07 & 0,61 \\
Curtosis Estandarizada & 0,35 & $-1,56$ & 0,14 \\
\hline
\end{tabular}

Fuente: Elaborada por los autores

A partir del procesamiento estadístico realizado se plantea que los datos poseen una distribución normal (ver Tablas 3 y 4); pues la mayor parte de los datos tienden a estar agrupados cerca de la media, y ninguno de los puntos incluidos se encuentra fuera de los límites de control, por lo que no se rechaza la hipótesis de que el proceso se encuentra en estado de control estadístico para un nivel de confianza del $95 \%$. En estos casos, el valor del sesgo estandarizado y la curtosis estandarizada se encuentra dentro del rango esperado $(-2 \mathrm{a}+2)$ para datos provenientes de una distribución normal; con una variación aceptable ya que a menor coeficiente de variación mayor homogeneidad en los valores obtenidos. El margen de error muestra un alto grado de confiabilidad de la precisión del ensayo realizado pues los mismos están por debajo del 2\%. Algo similar sucede con los contenidos de los ingredientes farmacéuticos activos (ver Tabla
2), pues la estabilidad de estos se mantuvo dentro de sus criterios de aceptación en el transcurso de 12 meses, aspectos que tributan a su actividad farmacológica, y que son indicativos de la seguridad y eficacia que posee el mismo durante su uso por parte del paciente, cuando se almacena a temperatura ambiente. En la evaluación realizada el lote $8001 \mathrm{~L}$ presentó un mejor comportamiento en el tiempo que el resto (ver Tabla 5) y en el caso del lote $8003 \mathrm{~L}$ en la valoración de cloruros, el valor de la curtosis estandarizada se encuentra fuera del rango $(-2,18)$, por lo que estadísticamente no se encuentra dentro del rango esperado para datos provenientes de una distribución normal; cambio que no resulta atribuible a la degradación del fármaco, ya que el contenido se mantuvo dentro de los criterios de aceptación establecidos, razón por lo que no se consideró significativo esta desviación.

Tabla 5.

Procesamiento estadístico de los contenidos de los principios farmacéuticos activos.

\begin{tabular}{|c|c|c|c|c|c|c|c|c|c|c|c|c|c|c|c|}
\hline Valoración & Sodio & & & Potasio & & & Citrato & & & Clorur & & & Dextro & & \\
\hline Lotes & $8001 \mathrm{~L}$ & $8002 \mathrm{~L}$ & $8003 \mathrm{~L}$ & $8001 \mathrm{~L}$ & $8002 \mathrm{~L}$ & $8003 \mathrm{~L}$ & $8001 \mathrm{~L}$ & $8002 \mathrm{~L}$ & $8003 \mathrm{~L}$ & $8001 \mathrm{~L}$ & $8002 \mathrm{~L}$ & $8003 \mathrm{~L}$ & $8001 \mathrm{~L}$ & $8002 \mathrm{~L}$ & $8003 \mathrm{~L}$ \\
\hline $\begin{array}{l}\text { Media de } \\
\text { proceso }\end{array}$ & 101,6 & 102,9 & 101,6 & 98,1 & 98,8 & 97, & 99,2 & 100,5 & 102,6 & 98,94 & 98,4 & 97,5 & 97,4 & 98,9 & 98,6 \\
\hline $\begin{array}{l}\text { Desviación } \\
\text { estándar } \\
\text { Coeficiente de }\end{array}$ & 2,30 & 1,46 & 1,95 & 2,26 & 2,26 & 2,93 & 1,77 & 3,28 & 3,24 & 1,62 & 2,68 & 2,69 & 0,58 & 1,35 & 1,46 \\
\hline $\begin{array}{l}\text { Variación } \\
(\%)\end{array}$ & 2,89 & 1,86 & 2,39 & 3,48 & 3,78 & 3,84 & 1,47 & 3,22 & 3,92 & 2,32 & 3,22 & 2,79 & 0,44 & 1,37 & 1,49 \\
\hline Error Estándar & 1,47 & 0,95 & 1,20 & 1,69 & 1,86 & 1,86 & 0,73 & 1,64 & 2,00 & 1,14 & 1,56 & 1,34 & 0,22 & 0,68 & 0,73 \\
\hline $\begin{array}{l}\text { Sesgo } \\
\text { Estandarizado }\end{array}$ & $-0,23$ & $-0,59$ & $-1,29$ & 1,07 & 0,18 & 1,49 & 0,81 & $-0,72$ & $-0,24$ & 0,24 & 1,34 & 0,0 & 0,97 & 0,47 & $-1,17$ \\
\hline $\begin{array}{l}\text { Curtosis } \\
\text { Estandarizada }\end{array}$ & $-0,91$ & $-0,59$ & 0,95 & 0,95 & $-1,04$ & 1,39 & 0,45 & 0,78 & $-0,76$ & $-1,66$ & 1,03 & $-2,18$ & 0,61 & 0,05 & 0,68 \\
\hline
\end{tabular}

Fuente: Elaborada por los autores 


\section{AMAZONIA \\ lvvestiga}

El control microbiano es una herramienta que se emplea en la identificación y cuantificación de microorganismos que garantiza que las materias primas utilizadas y el producto final fabricado cumplan con todas las especificaciones microbiológicas exigidas. En este estudio no hubo presencia ni crecimiento de hongos y bacterias, salmonella, escherichia coli,

Tabla 6.

Resultados de los ensayos físico-químicos, químicos y microbiológicos realizados a las Sales de Rehidratación Oral Osmolaridad reducida (solución oral) bajo las condiciones de almacenamiento propuestas.

\begin{tabular}{|c|c|c|c|c|}
\hline Índices & Lotes & Inicio & $\begin{array}{l}1 \text { h Temperatura Ambiente } \\
\text { debajo de }(30 \pm 2){ }^{0} \mathrm{C}\end{array}$ & $\begin{array}{l}24 \text { h en } \\
\text { Refrigeración } \\
(2-8){ }^{0} \mathrm{C}\end{array}$ \\
\hline \multirow{3}{*}{ pH $(7,0-8,8)$} & $8001 \mathrm{~L}$ & 8.3 & 8.2 & 8.3 \\
\hline & $8002 \mathrm{~L}$ & 8.2 & 8.2 & 8.3 \\
\hline & $8003 \mathrm{~L}$ & 8.2 & 8.2 & 8.3 \\
\hline $\begin{array}{l}\text { Formación de } \\
\text { precipitado }\end{array}$ & $\begin{array}{l}8001 \mathrm{~L} \\
8002 \mathrm{~L} \\
8003 \mathrm{~L}\end{array}$ & $\begin{array}{l}\text { No se forma } \\
\text { precipitado }\end{array}$ & No se forma precipitado & $\begin{array}{l}\text { No se forma } \\
\text { precipitado }\end{array}$ \\
\hline $\begin{array}{l}\text { Claridad de la } \\
\text { solución }\end{array}$ & $\begin{array}{l}8001 \mathrm{~L} \\
8002 \mathrm{~L}\end{array}$ & $\begin{array}{l}\text { Solución transparente } \\
\text { e incolora. No } \\
\text { presenta pintas ni } \\
\text { impurezas mecánicas }\end{array}$ & $\begin{array}{l}\text { Solución transparente e incolora. } \\
\text { No presenta pintas ni impurezas } \\
\text { mecánicas }\end{array}$ & $\begin{array}{l}\text { Solución } \\
\text { transparente e } \\
\text { incolora. No } \\
\text { presenta pintas ni } \\
\text { impurezas } \\
\text { mecánicas }\end{array}$ \\
\hline \multirow{3}{*}{$\begin{array}{l}\text { Conteo } \\
\text { Microbiano }\end{array}$} & $8001 \mathrm{~L}$ & Responde & Responde & Responde \\
\hline & $8002 \mathrm{~L}$ & Responde & Responde & Responde \\
\hline & $8003 \mathrm{~L}$ & Responde & Responde & Responde \\
\hline
\end{tabular}

Fuente: Elaborada por los autores

Según lo observado en la tabla 7 los datos provienen de una distribución normal, con un nivel de confianza del $95 \%$

Tabla 7.

Resultados estadísticos de $\mathrm{pH}$

\begin{tabular}{llll}
\hline Lotes & $\mathbf{8 0 0 1} \mathbf{L}$ & $\mathbf{8 0 0 2} \mathbf{L}$ & $\mathbf{8 0 0 3} \mathbf{L}$ \\
\hline Media de proceso & 8,27 & 8,23 & 8.23 \\
Desviación Estándar & 0,06 & 0,04 & 0,04 \\
Coeficiente de Variación (\%) & 0,70 & 0,70 & 0,70 \\
Error Estándar & 0,03 & 0,03 & 0,03 \\
Sesgo Estandarizado & $-1,22$ & 1,22 & 1,22 \\
\hline
\end{tabular}

Fuente: Elaborada por los autores

\section{Discusión}

Las características organolépticas de las Sales de Rehidratación Oral Osmolaridad reducida (polvo oral) permanecieron inalterables durante el tiempo de experimentación, lo que permitió establecer la idoneidad de las condiciones de almacenamiento propuestas, lo que es extensivo para el material de envase utilizado, el que garantiza la impermeabilidad del producto, evitando que el mismo interaccione física o químicamente con el medio ambiente, lo que puede originar fallas en el cumplimiento de los requisitos de seguridad, identidad y pureza durante su almacenamiento (Zunovic y Vranic, 2008; Lalitha, Sanjay, Vyshak y Kadiri, 2010).

El $\mathrm{pH}$ es una de las determinaciones analíticas más importantes utilizados en la química y bioquímica pues determina numerosas características notables de la estructura, de la actividad de las moléculas y del comportamiento de células y organismos. En una solución es uno de los factores que afecta la estabilidad y su valor 
debe permanecer cercano al de la mucosa gástrica para evitar problemas de tolerancia e irritabilidad (Fernández, 2019). Según se observa en la Tabla 2 los valores se presentan en un rango de 7,83 a 8,8 en todas las muestras con una variación no significativa en los resultados a lo largo del tiempo de estudio; comportamiento que se asemeja a los hallados para la pérdida por desecación, que se mantiene por debajo de $1 \%$.

Como se muestra en la tabla 6 los resultados obtenidos en el estudio realizado a la solución oral evidencian que durante el tiempo en que se mantiene la solución en conservación se garantiza el cumplimiento de sus características físicas y químicas, aspectos importantes para la estabilidad, ya que alteraciones significativas en el aspecto visual, $\mathrm{pH}$, y del contenido de las moléculas activas, son inadmisibles en estos estudios (Curti et al., 2019). La solución oral muestra también estabilidad microbiológica bajo las condiciones de almacenamiento declaradas. El análisis de estos parámetros en las condiciones de almacenamiento propuestas es esencial, teniendo en cuenta que numerosos medicamentos que son usados como soluciones o suspensiones orales reconstituidas deben ser almacenados en refrigeración, para garantizar la estabilidad química de los principios activos, así como para prevenir el crecimiento microbiano (Nwokoye, Oyetunde y Akinleye, 2012). En estos aspectos se ha profundizado en los antibióticos reconstituidos (Uzunović y Vranić, 2008; German, Bukowsska, Pajchel, Grzybowska y Tyski, 2010; Mieke, Alain, De Waele, \& Stove, 2016), sin embargo, no han sido suficientemente estudiados para las sales de rehidratación oral, por lo que consideramos que los resultados obtenidos en la presente investigación constituyen un valioso aporte en esta temática.

\section{Conclusiones}

Los ensayos realizados permitieron concluir que la formulación de Sales de Rehidratación Oral Osmolaridad reducida (polvo oral) mostró estabilidad en los 12 meses de estudio almacenada a $30 \pm 2{ }^{\circ} \mathrm{C}$ de temperatura y humedad relativa de $70 \pm 5 \%$ en sobres de papel aluminio-poliéster-polietileno por 20,5 g. La formulación de Sales de Rehidratación Oral Osmolaridad reducida (solución oral) mostró estabilidad almacenada en frascos transparente de vidrio (borosilicato) por una hora a temperatura ambiente $30 \pm 2^{0} \mathrm{C}$ y por 24 horas en Refrigeración $2-8^{0} \mathrm{C}$. Esta investigación permitió comprobar la adecuación de las condiciones de almacenamiento en el envase propuesto y definir el tiempo de vida útil de las Sales de Rehidratación Oral Osmolaridad reducida (polvo y solución oral), producto del gran interés social y económico que constituye un nuevo renglón exportable para $\mathrm{Cuba}$; siendo de gran importancia por la alta incidencia de enfermedades diarreicas agudas en los países tropicales.

\section{Referencias bibliográficas}

Blanco, M.A., \& Reyes, D. E. (2015). Análisis estadístico de la enfermedad diarreica aguda en pacientes atendidos en servicio de urgencias durante el 2012-2013. Revista Cubana de Investigaciones Biomédicas, 34(2), 104-111.

BP (2013). British pharmacopoeia 7 th edition. Her Majesty Stationary Office, Version electronica https://www.pharmacopoeia.com/

Curti, C., Harti, K. S., Lamy, E., Mathias, F., Bornet, C., Guinard, B., Fersing, C., Primas, N., Albanese, J., \& Vanelle, P. (2019). Stability studies of antipyocyanic beta-lactam antibiotics used in continuous infusion. Pharmazie 74, 357-362. doi: 10.1691/ph.2019.8215

Fernández, M. (2019). Maestría en Tecnología y Control de Medicamentos, diseño de medicamentos I. Departamento de Farmacia. La Habana, Cuba: Instituto de Farmacia y Alimentos, 1-25.

Galván, E. (2014). Epidemiología de las enfermedades diarreicas agudas. Cubana Med Gen Integr, 5(1), 48-68.

García, C. Montes de Oca, Y. Salomón, S. (2013). Control de la calidad y estudio de estabilidad del paracetamol gotas orales 100 mg/ml. Revista Cubana de Farmacia, 47(1).

German, R., Bukowsska, B., Pajchel, W., Grzybowska, G.y \& Tyski, S. (2010). Extremely long time stability study of selected antibiotic standars. Journal of pharmaceutical and biomedical analysis, 51(3), 758-763.

Granda, E. (2003). Rehidratación oral. Actuación inmediata. Revista Comité editorial Inicio Farmacia Profesional, 17(7) 43-47.

Herrera, I. F., García A. C., \& Mascareñas, A. H. (2018). Impacto de las enfermedades diarreicas agudas en América Latina. Revista Latinoamericana de Infectología Pediátrica. Sociedad latinoamericana de infectología pediátrica SLIPE, 31(1), 8-16.

Herrera, M. T., García, C. M., \& Barrios, M. A. (2008). Estabilidad de las gotas orales de picosulfato de sodio. Revista Cubana de 


\section{AMAZONIA \\ Dovestiga}

Farmacia, 42(2).

http://scielo.sld.cu/scielo.php?script=sci_artt ext\&pid=S0034

75152008000200004\&lng=es\&nrm=iso\&tln $\mathrm{g}=\mathrm{es}$

In, M., \& Miritello, R. (1988). Estabilidad de las sales de rehidratación oral en condiciones adversas de almacenamiento. Bol of Sanit Panam, 104(2), 197-200. https://iris.paho.org/handle/10665.2/17873

Ministerio de Salud del Perú (2010). Informe Técnico $\mathrm{N}^{\mathrm{o}} 03$ - 2010. Solución de Rehidratación Oral (Electrolitos). Decenio de las personas con discapacidad en el Perú, $\mathrm{pp}$

1-8.

https://studylib.es/doc/5089448/soluci\%C3

$\% \mathrm{~B} 3 n$-de-rehidrataci\%C3\%B3n-oral-electrolitos----digemid

Lalitha, N., Sanjay, P. N., Vyshak, M.G., \& Kadiri, U. (2010). Stability-indicating reverse phase HPLC method for the determination of cefazolin. Tropical Journal of Pharmaceutical Research, 9(1), 45-50.

Lemus, P. (2006). Análisis comparativo de estabilidad acelerada y estabilidad a largo plazo de Jarabe de Ambroxol en dos diferentes concentraciones, adulto y niños. (Tesis de pregrado). Universidad de San Carlos, facultad de ciencias químicas y farmacia. Escuela de química farmacéutica, Guatemala. Recuperado de http://biblioteca.usac.edu.gt/tesis/06/06_246 8.pdf

Materán, M R., Tomat, M., Salvatierra, A., León, K., \& Marcano, J. (2014). Terapia de rehidratacion oral en pacientes deshidratados por diarrea aguda infantil. Sociedad Venezolana de Puericultura y Pediatría, 77(1), $\quad$ 48-57. http://ve.scielo.org/scielo.php?script=sci_abs tract\&pid=S0004-

06492014000100009\&lng=pt\&nrm=iso\&tln $\mathrm{g}=\mathrm{es}$

Mieke, C., Alain, G.V., De Waele, J.J., \& Stove, V. (2016). Stability of amoxicillin and amoxicillin/clavulanic acid reconstituted in isotonic saline. Journal of Chemotherapy, 29(1).

DOI:

10.1179/1973947815Y.0000000052 http://dx.doi.org/10.1179/1973947815Y.000 0000052

Nwokoye, P., Oyetunde, O., \& Akinleye, M. (2012). Stability of reconstituted amoxicillin clavulanate potassium under simulated inhome storage conditions. Journal of Applied Pharmaceutical Science, 02 (01), 28-3.

OPS (2008). Organización Panamericana de la Salud. Manual de tratamiento de la diarrea en niños. Serie PALTEX para Ejecutores de Programas de Salud; 48. https://www.paho.org/journal/es

Pérez, R., Saíz, J., \& Pérez, L. (2009). Órgano de divulgación oficial Centro para el Control Estatal de la Calidad de los Medicamentos. Ámbito regulador. La Habana: Ministerio de Salud Pública de Cuba, edición ordinaria, 1-9. NÚMERO 00-100. ISSN 1684-1832

Pizarro-Torres, D. (2005). Alteraciones hidroelectrolíticas y ácido-base más frecuentes en el paciente con diarrea. Boletín Médico del Hospital Infantil de México, 62(1).

Povea, E. \& Hevia, D. (2019). La enfermedad diarreica aguda. Revista Cubana Pediátrica, 91(4).

http://www.revpediatria.sld.cu/index.php/pe d/article/view/928/467

Regulación No. 23-2000. Requerimientos de los estudios de estabilidad para el registro de productos farmacéuticos nuevos y conocidos. Ministerio de Salud Pública, https://www.cecmed.cu/reglamentacion/apro badas?page $=21$

UNICEF. (2001). Oral rehydration salts (ORS) A new reduced osmolarity formulation. https://rehydrate.org/ors/expertconsultation.html

USP 40. (2017). Farmacopea de los Estados Unidos de América. Ed. 40, (3), 6478 - 6481.

Uzunović, A., and Vranić, E. (2008) Stability of cefuroxime axetile oral suspension at different temperature storage conditions. Bosnian Journal of Basic Medical Science, 8, 93-97.

Vásquez, J., \& Arias, M. (2020). Informe anual de enfermedad diarreica aguda, hasta el periodo epidemiológico XIII. Dirección territorial de salud de Caldas, 1-18. http://observatorio.saluddecaldas.gov.co/des ca/anuales/2020/ENFERMEDAD\%20DIAR REICA\%20AGUDA.pdf

Zunovic, A., \& Vranic, E. (2008). Stability of cefuroxime axetil oral suspension at different temperature storage conditions. Bosnian Journal of Basic Medical Sciences, 8(1), 93-97. 